\title{
Stiftung für die berufliche Vorsorge der Leitenden Spitalärzte der Schweiz
}

\author{
U. Reinhard
}

In der Vergangenheit bestanden immer wieder Unklarheiten hinsichtlich der Anerkennung der steuerlichen Abzugsfähigkeit von Beiträgen, die Kaderärzte (Chefärztinnen und Chefärzte sowie Leitende Ärztinnen und Leitende Ärzte) an eine Vorsorgeeinrichtung der 2. Säule entrichteten. Im Juni 2005 hat sich die Schweizerische Steuerkonferenz (Vereinigung der schweizerischen Steuerbehörden, SSK) an einer Tagung mit dieser Problematik auseinandergesetzt. Dem VLSS ist es ein Anliegen, zur Klärung der Sachlage beizutragen und zukünftig Unsicherheiten zu vermeiden. Gerne möchten wir Ihnen deshalb den Beschluss der SSK darlegen und Ihnen zudem die Aufnahme in die Vorsorgestiftung des VLSS anbieten.

\section{Einkünfte aus ambulanter privat- ärztlicher Tätigkeit}

Die ambulante privatärztliche Tätigkeit des Spitalarztes wird vom Eidgenössischen Versicherungsgericht seit Jahren als selbständige Erwerbstätigkeit qualifiziert. Daran hat es trotz einzelner Ausnahmen [1] auch in jüngster Zeit festgehalten. Die Vorsorgestiftung VLSS ist die einzige Möglichkeit für die Versicherung dieser Einkommensteile, da dies nur beim Berufsverband möglich ist. Einzelanschlüsse an die Vorsorgeeinrichtung sind in allen Kantonen möglich. Bereits abgeschlossene Verträge behalten ihre Gültigkeit.

\section{Einkünfte aus stationärer privat- ärztlicher Tätigkeit}

Im Gegensatz zur angesprochenen «Sprechstunde», also der ambulanten privatärztlichen Tätigkeit, wurde die stationäre privatärztliche Tätigkeit von den Gerichten im Laufe der Zeit als unselbständige Erwerbstätigkeit aufgefasst, was sich inzwischen als konstante Rechtsprechung gefestigt hat [2]. Dies führte zu folgendem Problem: Kaderärztinnen und -ärzte konnten im Bereich der aus unselbständiger Tätigkeit erzielten Honorare nur bei der Vorsorgeeinrichtung ihres Arbeitgebers versichert werden. War das versicherbare Einkommen dort plafoniert, so konnten die Honorare nicht versichert werden: Einzelanschlüsse für die Versicherung dieses Einkommensteils bei einer anderen Vorsorgeeinrichtung waren nicht möglich. Damit waren Leitende Spitalärzte gegenüber anderen Berufsgattungen, die von Kadervorsorgen ihrer Arbeitgeber (sog. «Bel-étage-Versicherung») profitierten, klar benachteiligt.

Als Übergangslösung haben einige kantonale Steuerverwaltungen auch Honorare aus der stationären Behandlung von Halbprivat- und Privatpatienten, die eigentlich als Einkommen aus unselbständiger Erwerbstätigkeit gelten, steuerlich noch als selbständigen Erwerb gemäss Ziff. 1 hiervor weiter zum Abzug zugelassen.

Die SSK hat an ihrer Tagung nun offiziell Raum für eine einheitliche Lösung für alle Kaderärzte geschaffen. Für Honorare eines Kaderarztes, die AHV-rechtlich als Einkommen aus unselbständiger Erwerbstätigkeit qualifiziert werden und nicht bei der Basisvorsorge des Arbeitgebers versichert werden können, besteht die Möglichkeit, sich einer anderen Vorsorgeeinrichtung anzuschliessen und diesen Einkommensteil dort $\mathrm{zu}$ versichern. Diese Versicherung ist dann für alle betroffenen Ärzte des Spitals obligatorisch (Prinzip der Kollektivität und der Gleichbehandlung). Zudem muss der Arbeitgeber mindestens die Hälfte der Beiträge der Arbeitnehmer bezahlen. Schliesslich sind die Grundsätze der Planmässigkeit und der Angemessenheit zu gewährleisten.

\section{Die Lösung des VLSS}

Die Vorsorgestiftung des VLSS ermöglicht es Kaderärztinnen und -ärzten, die hiervor erwähnten Einkommensbestandteile zu versichern, welche durch die Plafonierung des Vorsorgereglements des Arbeitgebers dort nicht versichert werden können. Dabei werden die unversicherten Einkommensteile in einem einheitlich ausgestalteten Kollektivvertrag des Spitals im Rahmen der beruflichen Vorsorge versichert. Das Spital schliesst zu diesem Zweck für sämtliche am Spital tätigen Kaderärzte einen Anschlussvertrag mit der Vorsorgeeinrichtung VLSS ab. Es gilt spe- 
ziell zu vermerken, dass die Beiträge an die berufliche Vorsorge dabei steuerlich voll abzugsfähig sind: «Die aktuelle Lösung der Kadervorsorge der Vorsorgestiftung VLSS für leitende Spitalärzte kann daher steuerlich anerkannt werden.» [3]

Versichert ist derjenige Honoraranteil, welcher an die Kaderärzte ausgeschüttet wird. Es besteht die Möglichkeit, den ganzen Lohnbestandteil, welcher nicht in der Basisvorsorge versichert ist, über die Kadervorsorge VLSS zu versichern, oder auch nur einen bestimmten Anteil aus diesem Einkommen.

Da der Gesetzgeber vom Arbeitgeber eine mindestens 50\%ige Beteiligung an den Beiträgen verlangt, für das Spital aber nicht zusätzliche Kosten anfallen dürfen, muss der Arbeitgeberbeitrag vor der Festsetzung des an den Kaderarzt auszuzahlenden Honorars berücksichtigt werden. Dieser Aufwand gilt als Lohnnebenkosten und wird an die Kadervorsorge des Arztes überwiesen. Für das Spital besteht damit Kostenneutralität.
Mit dieser Lösung wird dem Vorsorgebedarf der Kaderärzte endlich Rechnung getragen und die jahrelange Ungleichbehandlung mit anderen Kadermitarbeitern aufgehoben. Für alle weiteren Fragen im Zusammenhang mit der Versicherbarkeit der Einkommensteile aus unselbständiger Erwerbstätigkeit des Spitalarztes wenden Sie sich bitte an die Geschäftsstelle der Vorsorgestiftung des VLSS: Pendia Associates AG, Tödistrasse 38, Postfach 2056, 8027 Zürich, Lotti Gautschi, Tel. 04428920 06, E-Mail: lotti.gautschi@pendia.ch. Zudem können Sie sich auch auf unserer Homepage www.vlss.ch detailliert über die Vorsorgestiftung VLSS informieren.

\section{Literatur}

1 Entscheid des EVG H 201/00 vom 19. März 2002; die Praxis des Bundesgerichts 1/1999.

2 BGE 122 V 281; BGE 124 V 97; EVG H 201/100 vom 19. März 2002.

3 Zitiert aus dem Beschluss der SSK vom 9. Juni 2005. 